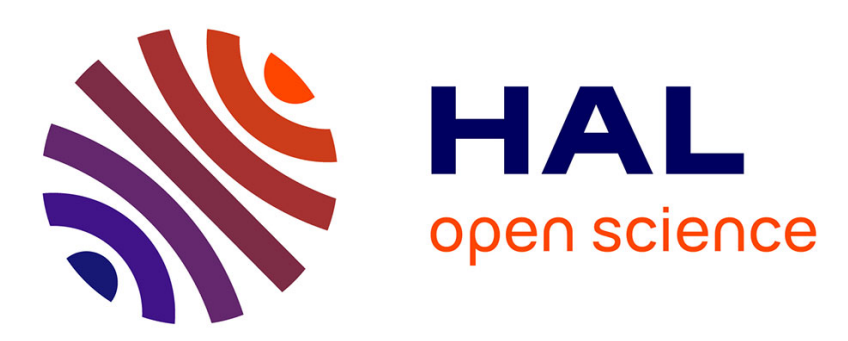

\title{
Mesure simultanée du pouvoir thermoélectrique et de la conductivité thermique de petits échantillons de 100 à $300 \mathrm{~K}$
}

\author{
D. Bideau, J.P. Troadec, J.L. Meury, G. Rosse, Dang Tran Quan
}

\section{- To cite this version:}

D. Bideau, J.P. Troadec, J.L. Meury, G. Rosse, Dang Tran Quan. Mesure simultanée du pouvoir thermoélectrique et de la conductivité thermique de petits échantillons de 100 à $300 \mathrm{~K}$. Revue de Physique Appliquée, 1978, 13 (8), pp.415-418. 10.1051/rphysap:01978001308041500 . jpa-00244469

HAL Id: jpa-00244469

https://hal.science/jpa-00244469

Submitted on 1 Jan 1978

HAL is a multi-disciplinary open access archive for the deposit and dissemination of scientific research documents, whether they are published or not. The documents may come from teaching and research institutions in France or abroad, or from public or private research centers.
L'archive ouverte pluridisciplinaire HAL, est destinée au dépôt et à la diffusion de documents scientifiques de niveau recherche, publiés ou non, émanant des établissements d'enseignement et de recherche français ou étrangers, des laboratoires publics ou privés. 


\title{
MESURE SIMULTANÉE DU POUVOIR THERMOÉLECTRIQUE ET DE LA CONDUCTIVITÉ THERMIQUE DE PETITS ÉCHANTILLONS DE 100 A $300 \mathrm{~K}$
}

\author{
D. BIDEAU, J. P. TROADEC, J. L. MEURY, G. ROSSE et DANG TRAN QUAN \\ Groupe d'Electronique et de Physique des Matériaux, \\ Université de Rennes, U.E.R. « S.P.M.», 35000 Rennes, France
}

(Reçu le 8 novembre 1977, révisé le 20 avril 1978, accepté le 8 mai 1978).

\begin{abstract}
Résumé. - Nous décrivons un dispositif permettant la mesure simultanée du pouvoir thermoélectrique et de la conductivité thermique de petits échantillons pour des températures allant de 100 à $300 \mathrm{~K}$. Le montage est testé sur le fer électrolytique SRM 734 conseillé par le NBS comme matériau de référence.
\end{abstract}

\begin{abstract}
An apparatus is described for simultaneous measurement of thermoelectric power and thermal conductivity of small samples over a temperature range 100-300 K. The SRM 734 electrolytic iron is used as standard reference material.
\end{abstract}

1. Introduction. - Le pouvoir thermoélectrique (PTE) $S$, et la conductivité thermique $\lambda$ d'un matériau sont habituellement déterminés séparément à l'aide de deux dispositifs distincts.

Le principe de mesure du PTE (méthode différentielle) consiste à créer entre les deux extrémités d'un échantillon une faible différence de température $\Delta T$ et à mesurer l'accroissement $\Delta E$ de la f.e.m. thermoélectrique qui en résulte. Le PTE est alors donné par

$$
S=\frac{\Delta E}{\Delta T}
$$

Pour la mesure de la conductivité thermique $\lambda$, les méthodes les plus employées font intervenir un flux de chaleur longitudinal $W$ tel que :

$$
W=-\lambda s \operatorname{grad} T
$$

où $s$ est la section de l'échantillon.

Le problème se réduit alors à la détermination d'un flux de chaleur, des dimensions géométriques de l'échantillon, et d'une différence de température.

2. Méthode de mesure. - 2.1 PouvoIr THERMoÉLECTRIQUE. - Nous utilisons la méthode décrite par Conan [1] (Fig. 1). Un thermocouple constitué de deux métaux $\mathrm{A}$ et $\mathrm{B}$ est placé en contact électrique et thermique avec chaque extrémité de l'échantillon $\mathrm{M}$.

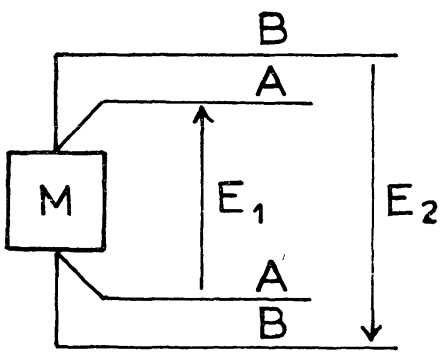

Fig. 1. - Principe de mesure du PTE.

[Schematic diagramm of apparatus for measuring TEP.]

L'une des extrémités est maintenue à température constante $T_{0}$. Un élément chauffant permet de faire varier lentement (régime de pseudo-équilibre) la température de l'autre extrémité; on mesure alors les variations $\Delta E_{1}$ et $\Delta E_{2}$ des f.e.m. $E_{1}$ entre les fils $\mathrm{A}$ et $E_{2}$ entre les fils B, et on en déduit le PTE du couple « échantillon M-métal A » par la relation

$$
S_{\mathrm{M} / \mathrm{A}}=\frac{\Delta E_{1}}{\Delta E_{1}+\Delta E_{2}} S_{\mathrm{B} / \mathrm{A}}
$$

$S_{\mathrm{B} / \mathrm{A}}$ étant le PTE du thermocouple de mesure.

2.2 Conductivité thermique. - En régime permanent, la puissance calorifique $W$ fournie par l'élément chauffant à l'échantillon provoque entre les 
extrémités de celui-ci une différence de température $\Delta T$ telle que :

$$
W=\lambda \frac{s}{l} \Delta T
$$

où $l$ est la longueur de l'échantillon.

Il apparait alors entre les fils A, dont l'association avec l'échantillon constitue un thermocouple différentiel, une tension $E_{1}$ telle que

$$
\Delta T=\frac{E_{1}-E_{1}^{0}}{S_{\mathrm{M} / \mathrm{A}}}
$$

$E_{1}^{0}$ étant la tension thermoélectrique en l'absence de chauffage.

La connaissance de $W$ et de $S_{\mathrm{M} / \mathrm{A}}$ suffit donc pour déterminer la conductivité thermique $\lambda[2]$ :

$$
\lambda=W \frac{l}{s} \frac{S_{\mathrm{M} / \mathrm{A}}}{E_{1}-E_{1}^{0}} .
$$

Cette technique permet ainsi de mesurer $S$ et $\lambda$ dans les mêmes conditions.

3. Dispositif expérimental. - 3.1 Cellule DE MESURE. - L'échantillon est placé entre deux électrodes constituées par des disques de cuivre (épaisseur $1,5 \mathrm{~mm}$, diamètre $10 \mathrm{~mm}$ ) usinés de façon à recevoir les fils de thermocouples (Fig. 2).

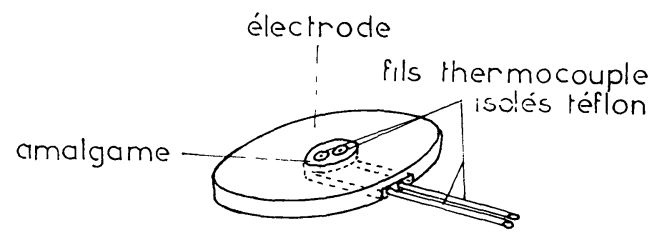

FIG. 2. - Détail des électrodes.

[Detail of plates.]

Ces fils (diamètre $2 / 10 \mathrm{~mm}$, gainés téflon) sont noyés dans leur logement au moyen d'un amalgame de très bonne conductivité thermique constitué de $66 \%$ de $\mathrm{Cu}$ et $34 \%$ de $\mathrm{Ga}$ [3]. Leur extrémité affleure la surface de l'électrode, assurant un contact quasiponctuel avec l'échantillon.

L'élément chauffant, fixé sur l'électrode supérieure, est constitué d'un anneau de carbone de résistance voisine de $5 \mathrm{k} \Omega$, noyé dans un cylindre de cuivre d'épaisseur $2 \mathrm{~mm}$ dont il est isolé par du vernis General Electric. Les fils d'alimentation $(\varnothing=2 / 10 \mathrm{~mm})$ sont en cuivre.

La cellule est placée sur l'échangeur d'un cryostat à circulation (MERIC C 6538) (Fig. 3). La régulation, commandée par une diode AsGa TG $100 \mathrm{~K}$, permet d'obtenir, au niveau de la partie supérieure de l'échangeur, une température constante (écart $<2 / 100 \mathrm{~K}$ ) pendant toute la durée de la mesure. Les dimensions de l'échangeur étant nettement plus importantes

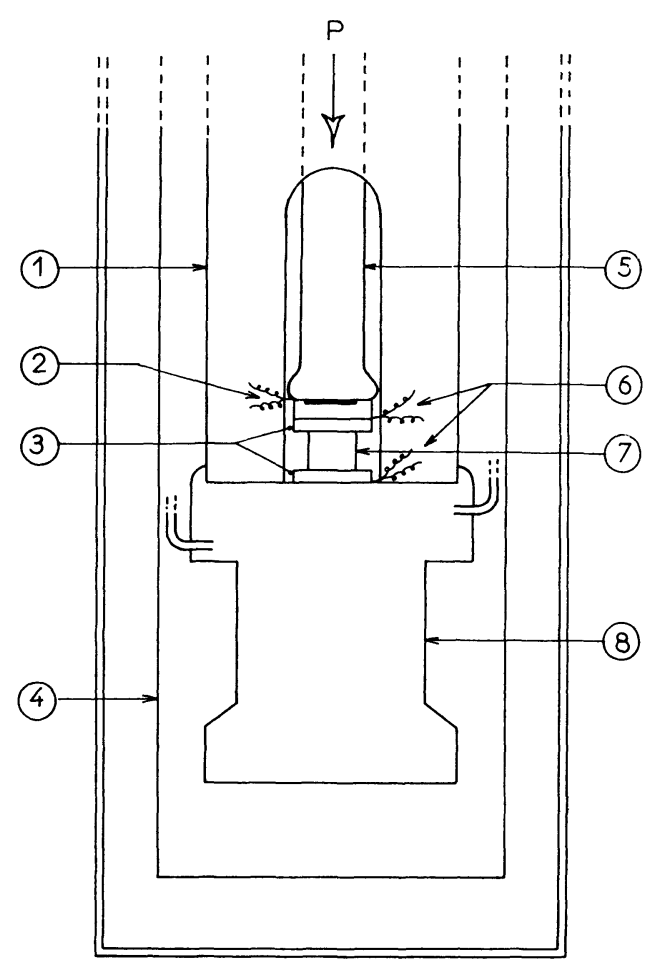

Fig. 3. - Cellule de mesure : 1. écran 1;2. élément chauffant ; 3. électrodes; 4. écran $2 ; 5$. piston de pyrex; 6. thermocouples; 7. échantillon; 8 . échangeur; $P$ : pression appliquée.

[Schematic drawing of apparatus : 1 . screen $1 ; 2$. hester ; 3. plates ; 4. screen $2 ; 5$. pyrex piston; 6. thermocouples; 7. sample; 8. copper thermal block; $P$ : applied pressure.]

que celles de la cellule, on peut prendre cette température comme température de référence.

Afin de réduire au maximum les résistances thermiques de contact, nous avons été amenés $[4,5]$

- à placer entre les éléments de la cellule une très mince feuille d'indium $(e<3 / 100 \mathrm{~mm})$;

- à appliquer sur l'ensemble, par l'intermédiaire d'un piston de pyrex (longueur $20 \mathrm{~cm}$, section $0,5 \mathrm{~cm}^{2}$ ) actionné par un dispositif hydraulique, une pression pouvant atteindre $60 \mathrm{~kg} / \mathrm{cm}^{2}$, maintenue constante pendant la manipulation.

3.2 Analyse du montage. - Dans le type de mesure envisagé, les grandeurs les plus difficiles à évaluer sont le flux thermique $W$ et la différence de température $\Delta T$.

D'une manière générale, les pertes thermiques qui limitent la précision de la détermination de $W$ sont de trois sortes : rayonnement, convection et conduction. Notre principe de mesure étant basé sur l'établissement de deux états successifs de pseudo-équilibre, seules les pertes liées à la mise en service de l'élément chauffant sont à considérer : les autres sont prises en compte par la mesure de la tension thermoélectrique $E_{1}^{0}$. Si nous nous limitons à des valeurs de $\Delta T$ inférieures à $0,5 \mathrm{~K}$, les pertes par rayonnement sont minimisées $(<500 \mu \mathrm{W})$ par l'utilisation d'un 
cryostat à double écran thermique $[5,6]$. Un vide poussé $\left(10^{-6} \mathrm{~mm} \mathrm{Hg}\right)$ limite considérablement les pertes par convection [6]. L'utilisation, pour l'alimentation de l'élément chauffant et pour les thermocouples, de fils fins $(\varnothing=2 / 10 \mathrm{~mm})$ et longs, thermalisés par bobinage autour de l'échangeur et de l'écran thermique intérieur permet de minimiser les pertes par conduction le long de ces fils dont la résistance thermique est ainsi de l'ordre de $4000 \mathrm{~W}^{-1}$.K. Enfin, il y a lieu de tenir compte des pertes par la partie supérieure de l'appareil (piston de pyrex de résistance thermique voisine de $4000 \mathrm{~W}^{-1}$. K).

L'erreur commise sur la détermination d'une température de surface résulte de la combinaison de trois effets [7]: un effet de macroconstriction dû à la convergence des lignes de flux dans la zone de contact échantillon-détecteur, un effet de microconstriction lié à l'existence de résistances de contact et un flux de fuite par le détecteur, dont il a été tenu compte au paragraphe précédent.

Dans notre montage, les effets de macro- et de microconstriction sont fortement réduits par l'utilisation de disques de contact (indium) et l'application d'une forte pression maintenue constante [5, 7]. Par ailleurs le positionnement des thermocouples au centre même du dispositif nous assure la meilleure configuration possible [8]. En réalité, l'erreur est encore réduite par le fait que nous mesurons non pas deux températures de surface, mais la variation de leur différence. Cette technique différentielle nous affranchit en outre des problèmes liés aux f.e.m. parasites dans la mesure où celles-ci restent constantes.

3.3 CirCUIT DE MESURE ET MODE OPÉRATOIRE. Les fils de thermocouples sont reliés, par l'intermédiaire d'une soudure froide maintenue à $0^{\circ} \mathrm{C}$, à un commutateur thermostaté TINSLEY $4092 \mathrm{~B}$. Les bornes communes de ce dernier sont connectées à un nanovoltmètre TEKELEC AIRTRONIC TE 925 utilisé en amplificateur. La tension ainsi obtenue est enregistrée en fonction du temps sur une table traçante SEFRAM TGM 164 (Fig. 4).

L'élément chauffant est alimenté par une source de tension continue régulée. La puissance qu'il dissipe est déterminée en mesurant la tension à ses bornes et le courant qui le traverse.

La technique expérimentale est la suivante :

a) Le système étant en équilibre thermique, on enregistre la tension $E_{1}^{0}$ en l'absence de chauffage ;

b) Lorsque l'élément chauffant est mis en service, on enregistre l'évolution des tensions $E_{1}$ et $E_{2}$; on en déduit le PTE $S_{\mathrm{M} / \mathrm{A}}$ de l'échantillon par rapport au matériau A ;

c) Lorsque le nouvel équilibre thermique est établi, on enregistre la valeur finale de la tension $E_{1}$; on peut alors, connaissant la puissance $W$ dissipée par l'élément chauffant, obtenir la conductivité thermique $\lambda$ de l'échantillon.

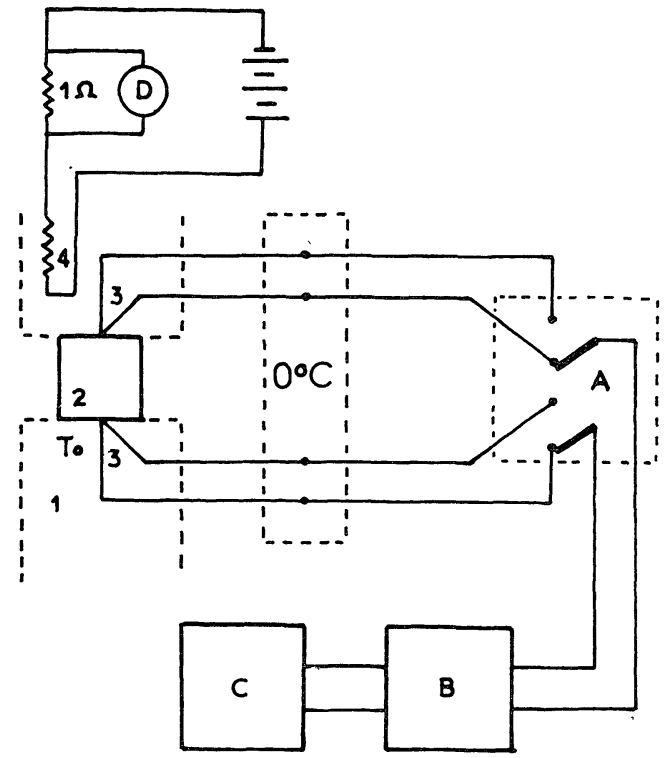

FIG. 4. - Circuit de mesure : 1. échangeur; 2. échantillon ; 3. thermocouples; 4. élément chauffant; A) commutateur : B) nanovoltmètre ; C) table traçante ; D) millivoltmètre.

[Block diagramm of equipment : 1 . isothermal block; 2. sample : 3. thermocouples; 4. hester; A) commutator; B) nanovoltmeter ; C) $X Y$ recorder ; D) millivoltmeter.]

4. Résultats expérimentaux. - Afin de tester notre dispositif expérimental, nous avons effectué des mesures sur le fer électrolytique SRM 734 dont le PTE est parfaitement connu [9], et qu'une récente étude du NBS [10] conseille comme élément de comparaison interlaboratoire pour les mesures de conductivité thermique. Pour nous placer dans nos conditions expérimentales habituelles, nous avons découpé un échantillon d'épaisseur $5 \mathrm{~mm}$ dans le barreau cylindrique $(\varnothing=6,4 \mathrm{~mm})$ qui nous a été fourni par le NBS.

Le polissage des faces de l'échantillon et l'application d'une pression supérieure à $30 \mathrm{~kg} / \mathrm{cm}^{2}$ se sont avérés nécessaires pour minimiser les résistances de contact et obtenir une bonne reproductibilité des résultats.

Nous avons utilisé des thermocouples cuivreconstantan qui présentent dans le domaine de températures exploré (100-300 K) une bonne sensibilité. L'examen des divers enregistrements a montré que les couples n'ont aucun retard entre eux et qu'aucune dérive due aux f.e.m. parasites n'apparaît.

La puissance dissipée dans l'élément chauffant, pour une différence de température $\Delta T<0,5 \mathrm{~K}$, est de l'ordre de $500 \mathrm{~mW}$.

La durée d'un point de mesure est inférieure à une heure.

A l'aide de la courbe d'étalonnage des thermocouples relevée in situ, on détermine le PTE $S_{\mathrm{M} / \mathrm{Cu}}$ de l'échantillon par rapport au cuivre. Le PTE absolu du cuivre étant connu [11], on déduit le PTE absolu du matériau.

$$
S_{\mathrm{M}}=S_{\mathrm{M} / \mathrm{Cu}}-S_{\mathrm{Cu}} \text {. }
$$




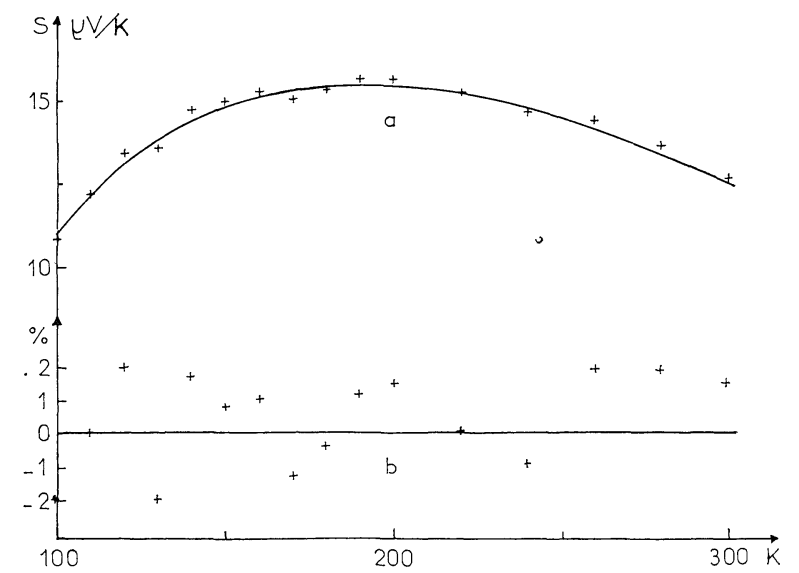

FIG. 5. - PTE du fer électrolytique SRM $734: a$ ) résultats expérimentaux :- Hust, + nos résultats ; $b$ ) écart.

[Temperature variation of the TEP of electrolytic iron SRM 734 : a) Experimental results :- Hust's results; + present results ; b) deviation.]

La figure $5 a$ présente nos résultats de mesure du PTE du fer électrolytique ainsi que ceux obtenus par Hust et al. [9]. L'écart entre ces résultats est inférieur à $2 \%$ dans toute la gamme de température explorée (Fig. 5b).

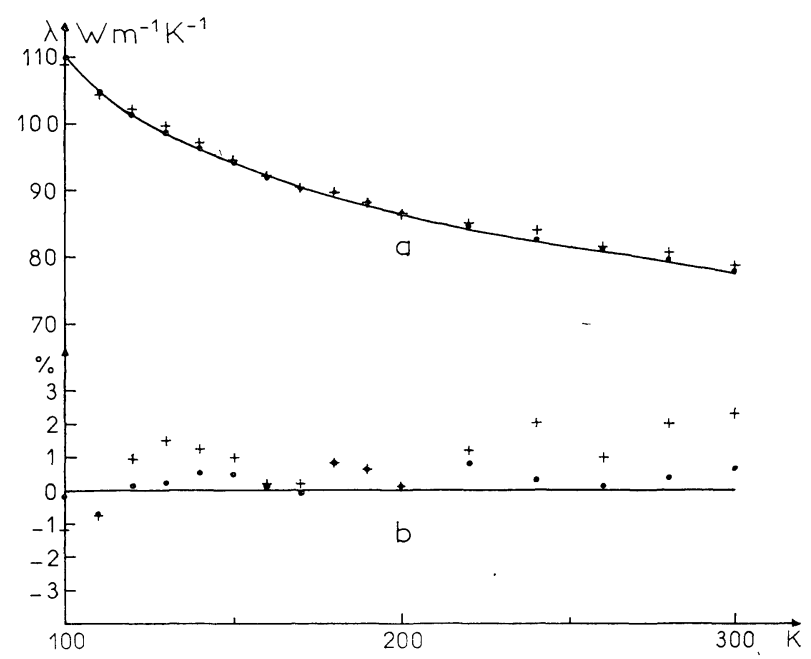

Fig. 6. - Conductivité thermique du fer électrolytique SRM 734 : a) résultats expérimentaux :- Hust ; nos résultats d'après les valeurs de PTE de Hust ; + nos résultats d'après nos valeurs de PTE $; b)$ écart.

[Temperature variation of the thermal conductivity of electrolytic iron SRM $734: a$ ) experimental results :- Hust's results; - present results from Hust's TEP values ; + present results from our TEP values $; b$ ) deviation.]
La figure $6 a$ donne les résultats des variations de la conductivité thermique obtenus :

1. A partir des valeurs du PTE données par Hust [9] ;

2. A partir de nos propres résultats de PTE;

3. Par le NBS (Hust et al. [9]).

L'écart entre nos résultats et ceux du NBS reste inférieur à $2 \%$ dans le cas 1 et à $3 \%$ dans le cas 2 dans toute la gamme de températures explorée (Fig. 6b).

Etant donnée la résistance thermique de l'échantillon les pertes par la partie supérieure de l'appareil sont ici négligeables.

5. Conclusion. - Le dispositif expérimental ainsi mis au point présente l'originalité :

- de permettre la mesure du PTE et de la conductivité thermique dans les mêmes conditions,

- d'être particulièrement adapté à l'étude d'échantillons de petites dimensions (quelques $\mathrm{mm}$ de diamètre et d'épaisseur).

La précision des mesures dépend essentiellement de la résistance thermique de l'échantillon étudié. $\mathrm{Si}$ cette résistance est supérieure à

$$
100 \mathrm{~W}^{-1} \cdot \mathrm{K}\left(\lambda<5 \times 10^{-3} \mathrm{~W}^{-1} \cdot \mathrm{cm}^{-1} \cdot \mathrm{K}^{-1}\right),
$$

les pertes par la partie supérieure de l'appareil occasionneront un gradient important dans l'échantillon dont la température ne sera plus bien définie.

La gamme de PTE couverte s'étend de quelques $\mu \mathrm{V} / \mathrm{K}$ à quelques centaines de $\mu \mathrm{V} / \mathrm{K}$.

Actuellement les mesures de conductivité thermique sont limitées, essentiellement par les pertes par conduction, à des échantillons de type métallique ou semiconducteur dégénéré

$$
\left(\lambda<5 \times 10^{-3} \mathrm{~W} \cdot \mathrm{cm}^{-1} \cdot \mathrm{K}^{-1}\right) .
$$

L'utilisation de fils plus fins (thermocouples et alimentation de l'élément chauffant) et d'un piston de résistance thermique plus élevée devrait permettre la mesure de plus faibles conductivités

$$
\left(\lambda<10^{-3} \mathrm{~W} \cdot \mathrm{cm}^{-1} \cdot \mathrm{K}^{-1}\right) \text {. }
$$

Les mesures réalisées couvrent présentement la gamme des températures allant de 100 à $300 \mathrm{~K}$. Des essais sont actuellement en cours pour étendre cette gamme, plus particulièrement vers les basses températures.

\section{Bibliographie}

[1] Conan. A., Goureaux. G. et Zoatrr. M.. Revue Phr. Appl. 6 (1971) 383.

[2] Weill, G., J. Phys. Rad. Phys. Appl. 23 Suppl. № 3 (1962) 15A.

[3] Harman, G. G., Rev. Sci. Instrum. 31 (1960) 7, 17.

[4] Laubitz, M. J., Therm. Cond., R. P. Tye (Academic Press) 1969, p. 110.

[5] Vuillermoz, P. L., Thèse, Lyon (1973).

[6] White, G. K., Therm. Cond., R. P. Tye (Academic Press) 1969, p. 69.
[7] BARdon, J. P., La mesure des températures de surface : Rencontre annuelle de la STF, La Baule, 1975, p. A 1.

[8] Maréchal, J. C., Mater. Constr. 7 No 37 (1974) 61.

[9] Hust, J. G. et Giarratano, P. J., Nat. Bur. Stand. Spec. Publ. 260 (1975) 50.

[10] Nat. Bur. Stand. Spec. Publ. 260, Ed. 75-76, 53.

[11] Nystrom, J., Ark. Mat. Astron. Fys. (1947) Band 34A No 27. 